\title{
PIERRE BAYARD: HACIA UNA CRÍTICA POLICIAL
}

\author{
Nicolás Garayalde \\ Universidad Nacional de Córdoba, Argentina - CONICET \\ nicolas_rio3@hotmail.com
}

Si la lectura es despojada de la ironía, pierde inmediatamente su carácter disciplinar y su capacidad de sorprender.

Harold Bloom, Cómo leer y por qué

RESUMEN / ABSTRACT

El siguiente artículo describe y problematiza la noción de crítica policial que podemos encontrar en un recorrido de la trilogía del autor francés Pierre Bayard compuesta por los libros Qui a tué Roger Ackroyd? (1998), Enquête sur Hamlet (2002) y L'affaire du chien des Baskerville (2008). Nos proponemos analizar el concepto de crítica policial con el objetivo de estudiar su impacto sobre conceptos propios de la teoría literaria como lectura, género, interpretación y marco de referencia, a los fines de problematizar la siguiente hipótesis: la crítica policial supone una operación interpretativa que lee una y otra vez la misma historia: la alegoría de la lectura.

Palabras ClaVE: crítica policial, lectura, género, marco de referencia, identidad.

The following paper describes and problematizes the notion of detective criticism that can be found in the trilogy by the French author Pierre Bayard which includes the books Qui a tué Roger Ackroyd? (1998), Enquête sur Hamlet (2002), and L'affaire du chien des Baskerville (2008). We are interested in analyzing the concept of detective criticism with the aim of studying its impact on concepts from literary theory such as reading, genre, interpretation, and frame of reference so that we can problematize the following hypothesis: detective criticism implies an interpretative operation which reads over and over the same story: the allegory of reading.

KEYWORDS: Detective Criticism, Reading, Genre, Frame of Reference, Identity. 


\section{LA TRILOGÍA}

¿Quién mató al padre de Hamlet? Es una pregunta que muchos no tardarían en responder, porque el texto escrito por Shakespeare parece explicitarlo sin demasiadas complicaciones. Una pregunta casi tan sencilla como esta otra: ¿Quién escribió Hamlet? Y sin embargo, ¿acaso Norman Holland no ha proclamado una vez, hace ya casi 40 años, que Hamlet era su mejor creación? ${ }^{1}$ Ahora bien, ¿es más sencillo responder la pregunta sobre el asesino del viejo rey de Dinamarca? El lector podría decir, sin dudar: ¡fue Claudio, el tío de Hamlet es el asesino! Pero, ¿estamos seguros? El crítico francés Pierre Bayard, en efecto, parece demostrar algo muy diferente en Enquête sur Hamlet (2002) cuando utilizando una serie de pruebas por momentos verdaderamente convincentes pone en cuestión la versión del espectro. Bayard se apoya en un elemento contundente, de difícil refutación: normalmente se dice que Claudio ha matado a su hermano para quedarse con su esposa. Se dice que el espectro del antiguo rey se aparece ante su hijo para pedir venganza. Se dice que Hamlet decide probar la culpabilidad de Claudio haciendo representar una obra que reproduzca, palmo a palmo, la modalidad con la que Claudio ha cometido el asesinato. Se dice que la reacción de Claudio frente a la obra demuestra su culpabilidad. Pero Pierre Bayard se pregunta, siguiendo una tradición que cuestiona esta lógica: ¿por qué Claudio no ha reaccionado ante la pantomima que se representa previamente y que narra exactamente la misma historia: la de un rey envenenado por la oreja? Hay otro elemento interesante en la argumentación de Bayard: ¿no parece inverosímil que existiese una obra, conocida ya por Hamlet, que contaba la historia tal y precisamente como ocurrió el extraño asesinato de su padre? ¿Ha escapado entonces el verdadero asesino? De ser el caso, ha logrado ocultar durante siglos su verdadera culpabilidad.

Pero no ha sido el único asesino impune de la literatura. Bayard ha bien sabido encontrar otros y volver a poner sobre la mesa preguntas hasta el momento obvias: ¿quién ha matado a Charles Baskerville, víctima en una de las últimas y más célebres aventuras de Sherlock Holmes? ¿Estamos tan seguros de que ha sido Stapleton, su vecino, ayudado por un enorme perro

Holland, Norman, "Hamlet - my greatest creation" (1975) Journal of the American Academy of Psychoanalysis 3: 419-427. Hay traducción al español en la antología de Holland que preparamos bajo el título Lectura, literatura y neuropsicoanálisis (Alción 2015). 
que esconde en el páramo de Dartmoor? ¿Y qué hay sobre aquel caso de Agatha Christie en la que Roger Ackroyd es asesinado por la espalda y el detective Poirot termina por señalar con su dedo inquisitivo al mismísimo narrador de la historia: el doctor Sheppard? ¿Ha sido él el asesino? ¿No se nos ha escapado, cegados por la arrogancia y la certeza psicótica de Poirot, quién efectivamente ha apuñalado a sangre fría en su oficina al señor Ackroyd?

Se trata de una interesante trilogía que ha escrito Pierre Bayard activando una ética de lectura que mira atentamente al texto para desenmascarar a los verdaderos asesinos. Se trata, sin más, de aquello que el mismo Bayard ha llamado crítica policial, que "apunta a ser más rigurosa que los detectives de la literatura y que los escritores y a elaborar soluciones que satisfagan más al espíritu". Una crítica "que interviene de manera activa, rechazando volverse cómplice" (L'affaire 68 - 69) 2 .

Por lo que Bayard no solo es un crítico, es además un detective. Pero allí no se detiene su ambición, porque la trilogía se incorpora dentro de un género que él mismo designa como teoría ficcional: el texto juega en la frontera entre la teoría y la ficción, utilizando a una como pretexto de la otra, haciendo teoría hacia y desde la literatura. La misma cualidad de este género híbrido es establecida en el primero de los tres libros cuando, desde el prólogo, Bayard afirma: "consagrado a releer una novela policial, este libro se ve, por la fuerza de las cosas, tener él mismo la forma de una novela policial" (Qui a tué 15).

La trilogía de Bayard, entonces, adquiere una homogeneidad al tratarse de novelas policiales sobre novelas policiales que caminan en el límite entre la literatura y la teoría. Para decirlo en pocas palabras: teoría ficcional policial. Pero el cruce de géneros no se detiene allí: Bayard es a su vez psicoanalista y la parte que toca a la teoría implica un ir y venir de la teoría literaria al psicoanálisis y de éste a la literatura. Los cruces entre psicoanálisis y literatura han sido una constante en el pensamiento de Bayard, quien ha llegado incluso a pensar esta relación en una dirección opuesta a la clásica cuando publicó, en 2004, Peut-on appliquer la littérature à la psychanalyse ?33

2 Todas las traducciones tanto del inglés como del francés en el presente ensayo me pertenecen, a menos que se indique lo contrario.

3 Hay traducción al español por Paidós: ¿Se puede aplicar la literatura al psicoanálisis? (2009). 
El objetivo del presente ensayo radica precisamente en una revisión de lo que Bayard ha llamado crítica policial. Esta revisión se detendrá en la trilogía compuesta por los libros Qui a tué Roger Ackroyd? (1998), Enquête sur Hamlet (2002) ${ }^{5}$ y L'affaire du chien des Baskerville (2008) ${ }^{6}$. Nos proponemos no solo analizar la noción de crítica policial sino además relevar su impacto sobre conceptos propios de la teoría literaria como lectura, género e interpretación.

\section{LA PREHISTORIA DE LA CRÍTICA POLICIAL}

El gesto fundante de la crítica policial radica en la lectura de sospecha que se conduce no a contrapelo del texto, en tanto no socava una verdad que el texto ocultaría, sino del detective. El texto, por sí solo, no ofrece una resolución u otra del enigma, aunque tradicionalmente la crítica se ha inclinado en favor del dictamen del detective (sobre todo en el policial clásico donde el detective es el equivalente a un hermeneuta de la razón). Es el texto mismo el que ofrece al crítico policial las herramientas para apuntalar una lectura que trastoque las bases lógicas que organizan el argumento del detective. En este sentido, la crítica policial de Bayard no es primera ni única, aunque sea su trilogía el campo específico donde se constituye y toma su forma más lograda.

En este sentido, es posible encontrar en la historia de la crítica literaria algunos textos que, al decir de Bayard (1998), supondrían esbozos de la posterior crítica policial. Entre ellos, quizás sea la crítica que Shoshana Felman (1983) hace respecto a Edipo Rey (2001) la que más se acerque a la constitución del género establecido por Bayard. No solo en la medida en que se trata de una lectura que cuestiona la resolución dada por el detective sino también por las consecuencias que su análisis tiene sobre las nociones de lectura e interpretación. No es casual que el mismo Bayard haya referido a este texto como formante de las condiciones de producción del género. Se trata del artículo que Felman publicó en 1983 en el número 42 de la revista Littérature bajo el título "De Sophocle à Japrisot (via Freud), ou pourquoi le

\footnotetext{
$4 \quad$ ¿Quién mató a Roger Ackroyd?

Investigación sobre Hamlet.

De la trilogía nombrada solo este último ensayo ha sido traducido al español por Anagrama bajo el título El caso del perro de los Baskerville (2012).
} 
policier ?", donde contrapone el Edipo Rey de Sófocles con la novela Piège pour Cendrillon de Sébastien Japrisot. Cuando la autora se aboca al análisis del Edipo Rey retoma una vieja protesta, presente ya en Voltaire, sobre la certeza de que haya sido realmente Edipo el asesino de Layo. La sospecha se sostiene sobre un elemento testimonial: uno de los testigos que estuvo en el momento del crimen de Layo afirma que fue un grupo de ladrones quienes mataron al entonces rey de Tebas en el cruce de los tres caminos. Ante esta información, la misma Yocasta siente aliviar su malestar frente a la posibilidad de haber copulado con su hijo y el testigo es llamado inmediatamente para su interrogatorio. Sin embargo, tal interrogatorio nunca sucede en cuanto es interrumpido por el testimonio del servidor que llevó de niño a Edipo a Corintios. Ante esto se resuelve para Edipo el enigma y la voz que contradice la resolución es simplemente olvidada. Así, dice Felman, "todo se explica, pero es precisamente la evidencia, la claridad, la 'luz' misma lo que es literalmente enceguecedora [littéralement aveuglante]" (37, el subrayado es mío).

La palabra aveuglante ofrece aquí cierta resistencia a su traducción en cuanto perdemos el doble sentido, contradictorio, que obtiene en francés. Que la luz sea literalmente aveuglante podría conducir a que en Edipo hay un pasaje al acto, la ceguera no es metafórica sino literal: Edipo se pincha los ojos y queda ciego frente a una ceguera previa, metafórica, que no le permite ver aquello que Tiresias, el ciego, ve. En este sentido es que tenemos la opción de traducir por literalmente enceguecedora en la medida en que es aveuglante littéralement, aveuglante en el sentido literal. Si, en cambio, optamos por el sentido no literal, figurado, de aveuglante, lo que obtendríamos sería literalmente evidente, como quien dice en francés la vérité est aveuglante ${ }^{7}$ (la verdad es evidente) ${ }^{8}$.

\footnotetext{
Ciertamente, el policial revela ciertos casos en que los dos sentidos juegan simultáneamente y llevan a conclusiones diferentes, como es el caso de "La carta robada" de E.A. Poe (1985). Es necesario que Dupin lea la carta (la lettre) literalmente aveuglante en un sentido figurado, es decir en su literalidad, à la lettre, allí donde la verdad es evidente. La policía, en cambio, que está ciega, no ve lo que está allí, está literalmente ciega. Que la verdad sea evidente implicará además, como veremos, la verdad como presencia, como lo que se manifiesta, lo que se descubre en evidencia, lo evideri: que aparece enteramente.

Para un lector familiarizado con la obra de Paul de Man, la relación entre visión y ceguera le parecerá aquí pertinente, en tanto ese doble juego está ya en acto en la lectura y un sentido se opone al otro sin dejar que el texto aloje una preferencia sin más empuje que el de la decisión. Véase Paul de Man (2010).
} 
Felman plantea que tanto en la novela de Japrisot como en el caso de Edipo Rey lo que vemos no es tanto el engaño en el que cae el asesino, como aquel en el que cae el detective. Se trata, dice, de la tragedia, del entrampamiento (piègemente) del detective. Pero Felman señala esta cuestión no tanto en el sentido en el que el detective es él mismo criminal, sino en el sentido en que su deseo mismo es subvertido, su deseo de intérprete, "el deseo de producir el fin de la investigación, es decir la verdad, la certeza del saber absoluto" $(38)^{9}$. La estructura interminable del relato hace que el detenimiento solo sea dado por el juicio, por el veredicto, que ocurre por fuera del enigma, en el exterior, siendo esta confusión su trampa allí donde Edipo investiga desde afuera el caso que ya lo contiene, que lo aloja en su interior ${ }^{10}$.

Cuando Felman habla del Edipo Rey como un policial sabe que está de algún modo transgrediendo la teoría clásica del policial que ubica los inicios del género en Poe. Precisamente por ello aclara que toma aquí al género policial como algo más extenso, basado en una cierta "estructura de los acontecimientos" y en una cierta "eficacia textual". Así, Edipo Rey implica una estructura de acontecimientos o eventos que se adecuan al esquema policial de la investigación de un crimen y de la revelación de un culpable. Está basado no tanto en el desarrollo de los personajes como en el desarrollo de la acción, y esa es la estructura a la que refiere Felman para decir que se trata de un relato policial. Hay, además, una puesta en escena de ese proceso de investigación, cosa propia del género. Aún más, estamos frente a una suspensión de la resolución, aquello que Roland Barthes ha llamado el retraso (le retard) dentro del código hermenéutico: "la verdad es rozada, perdida, desviada. Ese accidente es estructural"11 (S/Z 181).

$9 \quad$ Este deseo de verdad, de saber absoluto, sería precisamente el deseo que Jacques Derrida (1977) denuncia en la lectura que Lacan (2005) hace de "La carta robada". Como resume Babara Johnson: "El problema con la triangularidad psicoanalítica, a los ojos de Derrida, no es que contenga un número incorrecto de términos, sino que presupone la posibilidad de una mediación dialéctica exitosa y una normalización armoniosa o Aufhebung del deseo. Los tres términos en la tríada edípica entran en una oposición cuya resolución se parece al momento sintético de una dialéctica hegeliana" (472).

10 También Dupin juega en este exterior/interior del enigma allí donde para resolver el caso debe ingresar al sistema, ser portador de la carta, ocupar el lugar que antes ha ocupado el Ministro.

11 También la carta robada es perdida y desviada, suspendida, hasta llegar (o no) a destino. Y es también ese accidente estructural. 
Este procedimiento de suspensión y continuidad de la acción es comparado por Freud al psicoanálisis, según rescata Felman, y que aquí citamos de la edición de López-Ballester:

"La acción de la tragedia se halla constituida exclusivamente por el descubrimiento paulatino y retardado [verzögerten, la traducción de Felman es retardement] con supremo arte -proceso comparable al de un psicoanálisis- de que Edipo es el asesino de Layo y al mismo tiempo su hijo y el de Yocasta" (507).

Esto permite concluir a Felman que la misma estructura enigmática del policial puede alegorizar el enigma del inconsciente: "como el proceso de un psicoanálisis, la novela policial no es otra cosa que el relato del desplazamiento de la tarea ciega [aveugle] del intérprete: el relato de la autosubversión de la interpretación" (40). Este punto resulta de gran importancia porque expone precisamente el carácter autosubversivo, autodeconstructivo del relato, y la imposibilidad de cerrar un texto, de la interpretación como detenimiento artificial de un devenir del texto. Felman llama a este relato policial autosubversivo (en el que incluye también La interpretación de los sueños) el relato analítico [le récit analytique]. Felman dice que este es el relato de Japrisot, de Sófocles, de Freud ${ }^{12}$. Plantea así que el "relato analítico" más que una historia de la solución de un enigma es la historia de una lección del enigma. Se trata de un relato temporal del proceso de un suspenso, de una suspensión. Ese suspenso es lo que tiene en común, como dice Freud, el psicoanálisis con Edipo Rey. Pero ¿la suspensión de qué?

Felman propone que el relato policial toma como cuestión la interpretación, es decir, al lector mismo, puesto que se trata de un relato de desciframiento (de un enigma). De esta manera lo que pone en escena el relato policial es la propia interpretación y su carácter incompleto, en un sentido siempre diferido, retardado, desviado ${ }^{13}$.

Felman señala, entonces, las contradicciones que la obra de Sófocles tiene en torno a la resolución del mismo Edipo en tanto detective. En su afán pretendidamente exterior de resolver el enigma, el nuevo rey de Tebas cae en la trampa que está tendida en el interior y la ironía en juego radica en ser su propio perseguidor. 
De este modo, nos interesa subrayar una serie de elementos en el ensayo de la autora francesa que constituyen ya el germen de lo que nos parece central en el género de crítica policial para la teoría y crítica literaria. En primer lugar, el análisis sobre Edipo Rey que es llevado aquí plantea que se trataría en definitiva de una narración del problema de la interpretación. Es decir, según querríamos enunciarlo nosotros, de una alegoría de la lectura. En segundo lugar, este relato sobre lo que podría ser entendido en términos de entrampamiento del lector se sostendría sobre la base de una identificación del lector con el detective. Así las cosas, es el mismo lector el engañado allí donde se cree conducido a la verdad y no puede sino caer en una trampa. En tercer lugar, tendríamos también una equivalencia con el psicoanálisis, en la medida en que la interpretación está allí en juego, lo que abre la serie detective-lector-psicoanalista ${ }^{14}$. En cuarto lugar, el trabajo analítico sobre la obra de Sófocles revela la grieta por la cual el texto se autosubvierte en la medida en que la contradicción fisura la verdad del texto. Esta relación entre Edipo Rey y la verdad había sido ya trabajada por Michel Foucault en La verdad y las formas jurídicas (2000), donde plantea una lectura de la reconstrucción de la verdad por mitades. Foucault compara la forma de investigación de la verdad en la disputa Antíloco-Menelao que podemos leer en la Ilíada (donde a la verdad se llega por el juramento y lo que Foucault llama un "juego de prueba" y no acudiendo al testimonio de quien era el veedor de la competencia) con la forma de investigación en el Edipo. En la tragedia de Sófocles se da la reconstrucción de mitades donde el testimonio es crucial, el testimonio del servidor que lleva a Edipo a Corinitios, por ejemplo. Sin embargo, como el mismo Foucault lo señala al pasar, hay un testimonio, un fragmento, que es omitido, y es precisamente el del testigo del crimen, como se omite el testimonio del veedor de los juegos en la Ilíada. Yocasta alude a la cuestión de que se asesinó a Layo en el cruce de tres caminos por varios hombres. Y Edipo retiene de aquí solo lo de los tres caminos, como prueba que lo incrimina. Ahora bien, el mismo Foucault

14 Efectivamente, la lectura de Lacan de "La carta robada" se asienta sobre la hipótesis de que se trata de una alegoría del significante. El cuento vendría a narrar "la verdad que se desprende del momento del pensamiento freudiano que estudiamos, a saber, que es el orden simbólico el que es, para el sujeto, constituyente" (24, traducción de Tomás Segovia). La disputa con Derrida se apoyará sobre el estatuto de esta verdad y sobre la posibilidad de que, a fin de cuentas, la carta (la lettre) en cuanto significante, llegue a destino. Es decir, en la medida en que es posible una descifrabilidad del texto. 
habla de una verdad casi completa que se da en este acoplamiento entre el fragmento de verdad que posee Yocasta (fue en el cruce de tres caminos por varios hombres) y el que posee Edipo (asesiné a un hombre en el cruce de tres caminos). Señala entonces Foucault: "Y decimos que es casi completa porque falta aún un pequeño fragmento: saber si fue muerto por uno o varios individuos, cuestión que lamentablemente no se resuelve en la pieza" (44, traducción de Enrique Lynch).

El testimonio es un resto ${ }^{15} \mathrm{y}$ abre una fisura sobre el acoplamiento de mitades. Es precisamente esta fisura la que da lugar al movimiento de autosubversión que demanda Felman. Por lo que encontramos aquí en último término la operación principal (que deviene luego una problematización sobre la lectura misma) de señalamiento de la contradicción que atenta contra lo que el detective (en este caso el mismo Edipo) juzga como la verdad del enigma.

Sin embargo, la lectura de Felman se detiene allí y no avanza con alguna hipótesis que procure resolver el enigma. Ese es el gesto que fundamentará el género de crítica policial en la trilogía de Bayard, quien, como Dupin, decide intervenir para reconducir la carta desviada, perdida, hacia su destinatario ${ }^{16}$.

15 También hay un resto en "La carta robada". Un resto, primero, en la carta que el Ministro deja en lugar de aquella que roba a la Reina; un resto, luego, en la carta que Dupin deja en lugar de aquella que roba al Ministro. El primer resto está en lugar de una carta que se desvía, se pierde; el segundo en el lugar de una carta que vuelve a destino. Lo que la lectura de Lacan y la de Derrida tienen de diferencia radica precisamente, como ya hemos adelantado, en la cuestión de si la verdad es o no finalmente alcanzada. Dice Derrida: "En el momento en que el Seminario encuentra, como Dupin, la carta en donde ésta se halla -entre las piernas de la mujer-, el descifre del enigma ha anclado en la verdad. El sentido del cuento, el querer-decir de la carta robada ('lo que quiere decir la carta robada, incluso demorada, es que una carta siempre llega a destino'), sale a la luz. Descubrimiento de un querer decir (la verdad) hermenéutico, el descifre (el de Dupin, el del Seminario) llega asimismo a destino" (53-54, traducción de Hugo Acevedo). La posición de Derrida, en cambio, apunta contra la noción de indivisibilidad del significante y asienta su lectura sobre la diseminación.

16 La hipótesis que quisiésemos comenzar a avanzar aquí radicaría en la intervención del crítico-lector como necesidad, al igual que la intervención del detective o el psicoanalista. Que la carta llegue a destino no significaría, en este sentido, que la verdad sea desocultada, que el destinatario estaba ya prefijado, sino que, como señala Žižek (1994), la misma carta encuentra el lugar del destinatario. En palabras de Barbara Johnson: "El destino de la carta está así donde sea que es leída: el lugar que asigna a su lector como su propia parcialidad" (502). 


\section{HACIA UNA CRÍTICA POLICIAL: GÉNERO E INTERVENCIONISMO}

La categoría de crítica policial como género es establecida explícita y formalmente por Bayard en la tercera entrega de la trilogía L'affaire du chien des Barkerville. El elemento definitorio del género radica no tanto en el señalamiento de una contradicción, de un entrampamiento del detective, como en el gesto de una intervención. Esta operación supone posiciones epistemológicas en torno a la literatura. Para empezar, la intervención implica un trabajo crítico sobre un mundo incompleto, un "universo ahuecado" (univers troué), no por una pérdida sino por un "defecto/falta de estructura" (défaut de structure $)^{17}$. En este sentido, Bayard retoma una oposición, ya planteada en Qui a tué Roger Ackroyd?, entre "clausura textual" y "clausura subjetiva". La clausura textual remite a una clausura material, documental: en este sentido es que sería posible oponer la literatura a la historia. Si un hecho delictivo ocurrió en el mundo histórico y un nuevo documento ofrece evidencias de la inocencia de quien hasta entonces era considerado culpable estamos en todo derecho y deber de revisar el primer texto y de asumir las posibilidades de cambiar el veredicto. Pareciese, sin embargo, que la clausura textual del mundo literario teje sus límites más rigurosamente: "la realidad de una obra literaria -señala Bayard en este sentido- está estrictamente delimitada por los enunciados que la constituyen" (Qui a tué 126). Por supuesto, la misma idea de una "clausura subjetiva" comienza por agrietar esta separación tan clara y en el momento en que los mismos límites de la obra se fisuran, también lo hacen aquellos que separan literatura de historia. La pregunta que surge entonces aquí, para retomar el título de un libro de Barbara Johnson, sería: ¿cuál es el marco de referencia del texto? ${ }^{18} \mathrm{Si}$ es difícil delimitar el marco de referencia de un texto respecto a otros, aún más lo es si agregamos la

17 Poco necesitamos llamar aquí la atención sobre la carta de la Reina ante la pregunta: ¿qué contiene? O bien: ¿cuál es el significado de ese significante? El mismo Derrida, en su crítica a Lacan, señala aquí el lugar de un falocentrismo, en la medida en que la carta representa la falta, el falo que la Reina no posee, precisamente escondida bajo el hueco de la chimenea. Las expresiones de Bayard, aunque no remitan a Lacan explícitamente, se dejan leer a la perfección en un sentido lacaniano allí donde habla de "universo ahuecado" y "defecto/falta de estructura".

18 Efectivamente, es una pregunta pertinente en las lecturas que Lacan y Derrida hacen de "La carta robada". Una de las críticas que el último dirige al primero radica en el recorte que Lacan hace al omitir la trilogía en la que se inscribiría el cuento de Poe y descartar así del análisis los relatos "El doble asesinato" y "El misterio de Marie Roget”. Aún más, Lacan 
noción de "clausura subjetiva" en nuestras reflexiones al respecto, porque el universo del texto, ahuecado, en falta, supone zonas de entrada del universo del lector en la medida en que interviene en la elaboración del sentido. Así, el par exterior/interior del texto se ve cuestionado. Edipo Rey es ya la narración de esta disolución allí donde el detective pretendidamente exterior está involucrado sin saberlo en el interior. Para que una lectura tenga lugar es necesario atravesar la frontera e introducirse en el sistema. En un sentido que recuerda el espacio transicional de Donald Winnicott (1994), Bayard habla de un mundo intermediario, ni propio del texto ni propio del lector, un espacio fronterizo donde ocurrirían los intercambios posibles y donde la falta estructural de completitud del texto hallaría su concretización ${ }^{19}$.

Una posición epistemológica tal fundamentaría y justificaría un tipo de crítica intervencionista: "Por ello, el texto no es legible si el lector no le da su forma última, por ejemplo imaginando, consciente o inconscientemente, una multitud de detalles que no le son directamente proporcionados" (Qui a tué 127).

En la trilogía de Bayard se trata entonces primeramente de revelar las contradicciones del caso. Tomemos, por ejemplo, el análisis de la novela de Agatha Christie El asesinato de Roger Ackroyd. La investigación que lleva a cabo Poirot y su conclusión son conocidas. Roger Ackroyd aparece muerto en su despacho, apuñalado por la espalda, y el narrador, el doctor James Sheppard, es acusado del crimen. ¿Motivo? Sheppard sabía que la amante de Ackroyd, Mrs. Ferrars, había matado a su esposo y la chantajeaba bajo amenaza de denuncia. Tal situación terminaría por llevar a Mrs. Ferrars al suicidio dejando a su amante una carta con la revelación de la extorsión de Sheppard. Enterado de la situación el doctor se habría visto en la obligación de matar a Ackroyd. Pero ¿cómo cometer el crimen sin ser descubierto? La explicación de Poirot es la siguiente: el médico visita a su víctima y lo asesina en su despacho. Lleva consigo un dictáfono con el que registra la voz de Ackroyd mientras tienen una conversación. Luego de matarlo coloca un dispositivo que activará automáticamente el dictáfono de modo que los demás

no hace alusión tampoco al epígrafe de Séneca, lo que lleva a Derrida a preguntarse dónde empieza para Lacan el cuento de Poe.

19 El término concretización podría aludir a la teoría de la lectura de Wolfgang Iser, pero se encuentra lejos de compartir la misma perspectiva. En Iser la concretización está limitada por los bordes del texto y los "huecos de indeterminación" son espacios a completar por un lector textual al que Iser llama "lector implícito" (1984). 
habitantes de la casa lo escuchen cuando ya el Dr. Sheppard se ha retirado, suponiendo la hora de la muerte tiempo después de la visita del médico.

El primer movimiento de una crítica policial consiste aquí en subrayar las contradicciones propias de la solución del detective. Tomemos, por ejemplo, las inverosimilitudes ligadas al tiempo con el que cuenta Sheppard para organizar el asesinato entre la mañana (cuando se entera de la muerte de Mrs. Ferrars) y la tarde (cuando habría matado a Ackroyd). Bayard revisa todas las ocupaciones de Sheppard durante ese día y se pregunta: ¿en qué momento pudo haber diseñado el sofisticado aparato que activaría el dictáfono?

Bayard señala también inverosimilitudes ligadas al móvil. Plantea así que en realidad es poco viable que Sheppard haya intentado matar a Ackroyd porque desde el comienzo no representa un peligro real: la carta de Mrs. Ferrars se autoinculpa de asesinato también, por lo que no tiene demasiada autoridad. Pero además, ¿cómo sabría Sheppard que ella no envió otra carta a la policía? Matando a Ackroyd no solucionaría nada.

El trabajo de Bayard señalando estas incongruencias no se agota allí. Y solo nombramos aquellas que una economía sensata del espacio nos permite. Nos interesa en todo caso subrayar este primer movimiento propio de la crítica policial.

Frente a diversas evidencias que ponen en jaque la revelación del enigma dada por Poirot, el segundo movimiento es analizar oblicuamente el texto, leyendo los indicios a contrapelo del detective. El resultado es una operación de lectura que podríamos llamar irónica, donde al menos se revelan dos niveles posibles de sentido, excluyentes entre sí: la del detective y la del crítico policial. Este mecanismo supone un desdoblamiento clásico, propio del género, y que es complejizado aquí no solo en el modo en que Bayard lee la novela de Agatha Christie sino también por la identificación exacta narrador-criminal que es desarmada. Tal como señala Sergio Pastormerlo (1997), tradicionalmente la crítica ha planteado relaciones entre estos elementos que podrían formularse de la siguiente manera: "el narrador es al criminal lo que el lector es al detective" (34). La crítica policial operada por Bayard aquí mantiene esta equivalencia pero en un sentido muy diferente: en el momento en que el lector se distancia del detective denunciando su ceguera, aparta también al narrador del criminal. Por supuesto que lo que se nos quiere decir con esta ecuación no es que el narrador sea siempre, en el género, el criminal, sino que hay una equivalencia en cuanto a la ocultación de la verdad. Pero leída literalmente, la ecuación revela que la lectura del 
crítico francés establece un juego irónico que desdobla el texto y lo abre a su pluralidad.

Tomemos un ejemplo de esta operación doble de lectura a partir de una de las formas en que Sheppard evoca el último encuentro con Mrs. Ferrars: “¿Cuándo la había visto por última vez? No hacía más de una semana. Y tenía una actitud bastante normal considerando... las circunstancias" (Christie, The murder 11$)^{20}$. Para quien lee por primera vez la novela "las circunstancias" son aquellas que un lector puede ubicar como las propias de la víspera de un suicida. Para una relectura, "las circunstancias" son la extorsión de Sheppard sobre Mrs. Ferrars. Esta doble lectura, sin embargo, es propia del género policial, no de la crítica policial. En efecto, Bayard acuerda con Poirot en que Sheppard chantajeaba a Mrs. Ferrars, lo cual no significa que haya sido el asesino de Mr. Ackroyd. El hecho es que incluso en una lectura clásica que dé la razón al detective este fenómeno de doble lectura existe siempre y es la relectura la que lo evidencia. El primer efecto de esta afirmación es la consideración, contra una concepción común frente al género, de la relectura como necesaria. El mismo Pastormerlo ha problematizado esta cuestión en su diferenciación de la perspectiva restringida y la amplia frente al policial. Sin embargo, la operación de la crítica policial supone una relectura que pliegue la ironía y haga emerger un sentido que no es ni el de la lectura ni el de la relectura guiada por el detective. Veamos, por ejemplo, lo que Bayard llama mentira por omisión. En su relato, Sheppard dice la verdad, pero no toda la verdad. De este modo, al salir de su entrevista con Mr. Ackroyd nos relata:

$20 \quad$ La versión en inglés dice: "When had I last seen her? Not for over a week. Her manner then had been normal enough considering - well considering everything". La traducción francesa que cita Bayard es la siguiente: "Quand l'avais-je vue vivante pour la dernière foi ? La semaine précédente et son attitude avait été normale étant donné... les circonstances" (citado en Bayard, Qui a tué 54). He elegido atenerme a una traducción inglesa para la primera parte y optar por traducir, luego de los puntos suspensivos, a la par de la versión francesa dada por Bayard, de modo que se comprenda mejor el análisis que el crítico lleva a cabo. De todas maneras, la traducción más apegada al inglés no alteraría la lectura que hace Bayard, allí donde podríamos haber traducido de la siguiente manera luego de los puntos suspensivos: “...bueno, considerando todo el asunto". Debemos señalar, en todo caso, que en algunas traducciones de esta novela de Agatha Christie, como la editada por Planeta a cargo de G. Bermard de Ferrer (2007) se omite el pasaje que va luego del guión en la edición inglesa. Valga el aviso para el lector de este ensayo que tenga en sus manos tal edición y no pueda constatar el argumento de Bayard. 
Le habían traído la carta a las nueve menos veinte. Eran las nueve menos diez cuando lo dejé, con la carta aún sin leer. Durante un momento dudé mientras sostenía el picaporte con la mano, mirando hacia atrás y preguntándome si había dejado algo sin hacer. Nada vino a mi mente. Con un meneo de cabeza salí y cerré la puerta tras de mí (Christie, The murder 37).

Cuando, hacia el final de la novela, Sheppard confiesa ser el asesino ${ }^{21}$, vuelve precisamente a este pasaje, felicitándose por la ironía que expresa. El doctor no miente, el relato es exacto. Aún más, nos sugiere imaginar que ha puesto una línea de puntos luego de la tercera oración: "¿Se habría preguntado alguien qué hice en esos diez minutos?". Todo el pasaje inicial invita a diferentes lecturas. La primera de ellas, sin la revelación de Poirot y la confesión de Sheppard, no da demasiada importancia ni a la vacilación ni al olvido de algo que podría haber dejado sin hacer. Una relectura guiada por el detective otorga un sentido diferente a este "dejado algo sin hacer": se trata de un momento de vacilación en el que el asesino calcula si todos los elementos de su coartada han sido bien colocados y si ningún detalle se le ha escapado. El meneo de la cabeza es simplemente la negación de que algo quede sin hacer, pues nada acude a su cabeza. La relectura de la crítica policial, en cambio, ofrece otro sentido al pasaje: dejar algo sin hacer es matar a Mr. Ackroyd, porque Sheppard sabe que está a punto de leer la carta que lo incrimina como el chantajista de Mrs. Ferrars ${ }^{22}$. Pero el hecho de que nada

21 Efectivamente, luego de la acusación de Poirot, Sheppard admite ser culpable. Sin embargo, Bayard señala que el médico toma el lugar del verdadero asesino, su hermana Caroline, para protegerla.

22 Ciertamente, sabemos que la carta denuncia al chantajista. Pero nunca accedemos verdaderamente al nombre que la carta da, porque Ackroyd solo lee la mitad y se detiene; luego, finalmente, la carta es sustraída del despacho de la víctima. Cuando Sheppard abandona el despacho deja a Ackroyd "con la carta aún sin leer". En la explicación de Poirot tal lectura nunca se lleva a cabo, sencillamente porque Ackroyd es asesinado antes; en la versión de Bayard, la lectura de la carta permanece indecidible porque no podemos saber si ha ocurrido o no antes de que Caroline entre al despacho. Lo que resulta cierto, en todo caso, es que, como en "La carta robada", podemos advertir aquí una relación triangular en torno al significante: el mismo Bayard analiza la relación diádica madre-hijo que mantienen el doctor y su hermana a quien se adhiere, completando el triángulo, el detective Poirot como lugar de la ley. El destino de la carta se vuelve a su vez también indecidible, porque tanto Poirot como Bayard ofrecen destinaciones diferentes y uno podría afirmar sin demasiado preámbulo que el destinatario está allí donde la carta ha llegado. 
acuda a su mente junto al meneo de la cabeza implican que Sheppard, cuya psicología Bayard analiza de modo que parece más coherente dentro de esta decisión, termina por optar por retirarse, descartando la idea.

Como puede advertirse, las tres lecturas son válidas aunque se excluyen mutuamente, lo que da absoluto sentido a las siguientes palabras de Bayard: "cada libro cuenta, más allá de la anécdota policial, la misma historia repetida una y otra vez: la ceguera de quien lee" (Qui a tué 36). Si tomamos las dos concepciones frente al género que describe Pastormerlo podemos observar el modo en que la crítica policial se adecuaría a una visión amplia: "Una concepción restricta del policial pregunta, retóricamente: si los textos policiales se agotan en su final, ¿cómo es posible la relectura? Una concepción amplia podría responder con otra pregunta, también retórica: si todos los textos policiales son iguales, ¿cómo es posible la lectura en serie?” (31). En este sentido, Bayard podría acordar con el título del artículo de Edmund Wilson (1967) “¿A quién le importa quién mató a Roger Ackroyd?” (aunque no con su contenido) porque, en efecto, no es la revelación del criminal lo que verdaderamente está en juego en el libro de Bayard, sino la narración del modo en que un texto es "una máquina de producir lo indecidible" (Qui a tué 79). Pero el movimiento intervencionista, además, apunta a las posibilidades de la crítica como modalidad de escritura: "No es sorprendente - dice Pastormerlo hablando de la perspectiva amplia- que muchos escritores figuren entre los mejores lectores de este tipo, ya que este modo de lectura es una lectura de escritor. Leer el género como abstracción es leer sus posibilidades, es decir, sus posibles usos" (28).

Lo señalado hasta aquí nos permite entonces afirmar que la crítica policial involucra una perspectiva amplia del género que procura leer una y otra vez la misma historia: la alegoría de la lectura. Es decir, el relato del modo en que la lectura es aveuglante: ciega y evidente a la vez.

\section{LO QUE SUCEDIÓ EN EL PÁRAMO DE DARTMOOR: EL MARCO DE REFERENCIA}

Tal como el mismo Dr. Sheppard lo señala hacia el final de la novela, siempre es posible agregar algo que ha sido omitido entre dos oraciones. Lo que nos devuelve a la pregunta que ya habíamos anunciado retomando el libro de Barbara Johnson: ¿cuál es el marco de referencia? El problema del marco es problematizado también por la crítica policial, en tanto la intervención 
depende en gran medida de sus límites. Otra manera de formular esta cuestión sería la siguiente: ¿qué se puede incluir o excluir en el texto? O bien: ¿cuáles son los límites hasta donde podemos buscar los indicios del crimen?

Las fronteras son tanto internas como externas. Como se ha visto en el caso de la novela de Agatha Christie, el texto está plagado de lugares que pueden ser completados por el lector y no dichos por el texto, lugares que fisuran la propia homogeneidad interna. Por otro lado, tenemos los límites externos del texto tanto en relación con otros textos como con su vínculo con la realidad.

El asesinato de Roger Akcroyd ha ejemplificado la malla agrietada intratextual. Veamos ahora lo que toca a las relaciones externas. Podemos acudir, para ello, a la tercera entrega de la trilogía de Bayard, abocada esta vez al caso de El sabueso de los Baskerville (Conan Doyle, 1994), cuya historia es conocida: Charles Baskerville, adinerado señor que habita en la zona de Devonshire, es encontrado muerto en el sendero del páramo de Dartmoor. ¿Causa? Un ataque al corazón. Sin embargo, el doctor Mortimer, médico personal de Charles, acude a la casa de Sherlock Holmes frente a lo que considera algunos indicios enigmáticos que rozan lo fantástico: una vieja leyenda de un perro que acecha el linaje de Baskerville; las huellas desproporcionadamente grandes de un can en las cercanías del cadáver; el testimonio de algunos lugareños de haber visto una bestia de lengua y ojos brillantes rondar el páramo.

¿Qué contradicciones encuentra aquí Bayard? Detengámonos en una de ellas: sabido es que la conclusión de Holmes apunta a un enorme mastín entrenado por Stapleton, vecino y amigo de Charles Baskerville, que al enterarse de que es pariente de este último decide asesinarlo para tomar la posesión de sus bienes. Nadie, ni siquiera Charles, conoce esta relación. Stapleton, conociendo la fragilidad del corazón de su lejano familiar, decide revivir un antiguo mito, adquirir un gigantesco perro amaestrado y acondicionado y generar las circunstancias adecuadas para que un infarto se convierta en el arma de sus planes. Sin embargo, muerto Charles, aparece recién llegado desde Canadá un sobrino desconocido del linaje de los Baskerville: Henry. Por lo que el plan de Stapleton obliga al perro a volver a la acción. La perspectiva de Bayard comienza por denunciar lo absurdo de la hipótesis de Holmes, que es obligado a dar vueltas rocambolescas para explicar las ridículas ideas que Stapleton habría tenido para llevar a buen término su plan. Uno de los problemas principales proviene no tanto de cómo lograr que los ataques del perro lleguen a buen fin sino de cómo cobrar la herencia. Es una cuestión 
que el ingenio de Watson, siempre vilipendiado por su amigo detective, no deja de señalar hacia el final:

- Solo resta una dificultad más: si Stapleton lograba tener la herencia, ¿cómo explicar que él, el heredero, hubiese podido permanecer en el anonimato y vivido tan cerca de la mansión? -pregunté.

- Es muy compleja esa situación y me temo que es pedir demasiado que le resuelva su duda. El pasado y el presente están dentro de los límites de la investigación, pero es muy difícil contestar la pregunta de qué cosas puede hacer un hombre en el futuro (Conan Doyle, $E l$ sabueso 188, traducción de Juan Izquierdo).

A lo largo del análisis de la novela vemos un Bayard crítico que desanuda cada una de las contradicciones que, otra vez por economía de espacio, nos vemos aquí obligados a omitir. Seguidamente, como es lógico en la crítica policial, decide poner al verdadero criminal al descubierto: nos enteramos entonces de que Stapleton ha sido desde el inicio víctima de un largo y cuidadoso plan elaborado por su mujer, Beryl.

Sin embargo, la misma hipótesis de Bayard es cuestionada por una novela, posterior en su publicación, del autor francés Fabien Grégory, titulada Ce chien de Sherlock Holmes! (2014) ${ }^{23}$. Independientemente de que Grégory proponga otra versión de los hechos, apuntando a otro asesino, ni a Stapleton ni a Beryl, lo que resulta interesante es que lo hace desde una obra ficcional. Pero aún más, se trata de una novela que pone en jaque el mismo marco de referencia del texto desde su estructura. La obra está dividida en cinco partes: 1) un testamento firmado por un tal John Williams, habitante de Coombe Tracey y vecino de Charles Baskerville, explicando la existencia de un manuscrito de su autoría donde cuenta lo que verdaderamente sucedió a Mr. Baskerville; 2) una nota aclaratoria que cumple la función de macrorrelato con un metanarrador homónimo al autor de la obra donde se cuenta el hallazgo, en 1997, del manuscrito en el cementerio de Coombre Tracey; 3) El sabueso de los Baskerville, traducido al francés por A. de Jassaud; 4) el testamento de John Williams; 5) un epílogo del metanarrador. El conjunto de estos elementos constituye la novela de Ce chien de Sherlock Holmes! que incluye en su interior, como parte constitutiva, la obra de Conan Doyle. En 
este sentido, lo primero que es posible advertir es el modo en que la frontera entre los textos se diluye y el marco de referencia se trastoca. ¿Es legítimo considerar los elementos dados por John Williams para leer el relato de Watson? ¿Deberíamos incluir en el marco de referencia la obra de Fabien Grégory? Si ya polémica, esta ampliación del marco de referencia tiene la salvedad, todavía, de involucrar dos obras ficcionales. Sin embargo, el epílogo del macrorrelato alude explícitamente a Pierre Bayard y su historia sobre lo que pasó en el caso del perro de los Baskerville: "Será necesario así desmontar dos bellos edificios, teorías que acuden a explicar el caso del perro de los Baskerville: aquella de Watson y aquella de Pierre Bayard" (Grégory 314). Y luego, apenas más adelante, en alusión al manuscrito de John Williams: "¿Inventó toda esta historia? ¿La vivió? ¿Son valederos sus argumentos? No lo sé. (...) Por mi parte, ya no logro distinguir lo real de la ficción, lo verdadero de lo falso" (Grégory 316). En efecto, la frontera se ha vuelto difusa también entre la ficción y la realidad. A la vez, sin embargo, la misma obra de Bayard podría considerarse ficción: él mismo acaba por señalar que la crítica policial, a fuerza de actuar como detective, termina por reescribir una novela policial.

De un modo u otro, se trata de lo que en L'affaire du chien des Baskerville se llama una perspectiva integracionista de la relación ficción-realidad frente a una perspectiva segregacionista. Así, esta última establecería un límite claro entre realidad y ficción sin relación de continuidad entre una y otra; la integracionista, en cambio, supondría fronteras difusas y posibilidades de migración entre un mundo y el otro. Dos argumentos fundamentales sostienen esta última posición, que Bayard no tarda en tomar: 1) lingüístico: "el lenguaje no permite hacer la separación entre los seres reales y los personajes imaginarios y la integración de estos es entonces inevitable, se tenga el espíritu abierto o no" (L'affaire 117); 2) psicológico: "los personajes quizás no tienen realidad material, pero tienen una realidad psicológica, y esta conduce, se quiera o no, a una forma de existencia" (L'affaire 118 - 119). El inconsciente no conoce límites entre ficción y realidad y Emma Bovary o el Quijote han sabido demostrar esto ${ }^{24}$.

$24 \quad$ Este argumento psicoanalítico ha sido reforzado últimamente por la neurología y el modo en que explican el estado de absorción en el que una persona entra al leer una novela o mirar una película. Puede consultarse al respecto el artículo de Norman Holland "The Willing Suspension of Disbelief: A Neuro-Psychoanalytic View" (2003) que será editado en español en la antología referida más arriba en la nota 1 . 
En efecto, Bayard emplea la perspectiva integracionista para explicarse por qué Conan Doyle ha escrito una novela en la que Holmes comete tales equivocaciones: el escritor, fatigado del género, anhelaba hacía tiempo dedicarse a la literatura fantástica, razón por la cual decidió narrar la muerte de Holmes durante una de sus últimas aventuras. La reacción del público fue tenaz y Conan Doyle se vio obligado a volver sobre las aventuras de Sherlock Holmes con El sabueso de los Baskerville. Imposibilitado de matarlo, su venganza inconsciente, dice Bayard, se articuló en torno a la narración de una historia en la que se expusiera el error de la lógica de hierro del detective londinense.

Juan José Saer ha ido mucho más lejos, si hemos de poner un ojo crítico policial a su relato "Recepción en Baker Street" (2012), donde Tomatis cuenta entre cervezas una nueva aventura de Sherlock Holmes que piensa escribir en verso para alinearla a la tradición policial que inaugura el Edipo Rey. El caso es el siguiente: en un hospital de Inglaterra una enfermera envenena dieciséis recién nacidos y luego se suicida empleando el mismo método. Holmes, ya viejo, resuelve el caso desde su domicilio frente a Watson, un inspector joven y Lestrade, un jubilado de Scotland Yard. Un dato parece remarcable en el análisis de Holmes: solo quince de los dieciséis niños estaban registrados. Según el legendario detective, la enfermera habría llegado a trabajar en la maternidad por recomendación de Lord W., "miembro hereditario de la cámara de los Lores, que había sido uno de los principales benefactores de la maternidad" (Saer 95). Tiempo atrás, la enfermera se habría hecho cargo de Lady M., miembro de la familia del Lord, durante el tiempo que sufrió una larga enfermedad. Holmes asume que durante ese tiempo el contacto con el Lord W. habría devenido en una relación cuya consecuencia fue el embarazo de la enfermera. El bastardo, rechazado por Lord W, habría sido ocultado por la mujer en la maternidad bajo el temor de que sea asesinado por su padre biológico. Lord W., enterado de esta situación, pero ignorante de cuál sería el bebé correspondiente, decide envenenar a la mujer y a todos los niños recordando el episodio bíblico de Herodes. Los elementos con los que cuenta el detective son entonces los siguientes: 1) hay un recién nacido no registrado; 2) hubo una presunta relación entre la enfermera y Lord W. con un embarazo que se deduce de "un par de descomposturas" de la mujer en la maternidad. Por supuesto, los elementos no son suficientes, por lo que la estrategia de Holmes pasa por demostrar su hipótesis a la luz de "ciertos 
acontecimientos que no se han producido todavía"25 (95). Para ello solicita ayuda a un tal Danny el Rata, que le estaría debiendo un favor a Holmes: ladrón perfeccionista que habría sido culpado de un triple crimen cometido en Leeds del que sería inocente. Holmes habría demostrado que la Rata se hallaba en el momento del crimen "en Cornuailles desvalijando una mansión burguesa" (102) donde el detallista ladrón no habría dejado huellas. La tarea de la Rata habría sido ahora esconder sigilosamente en el departamento de la enfermera un falso telegrama, con fecha de mes y medio atrás, que sugiriese que el "documento revelador está en buenas manos" indicando la dirección de Holmes. La llegada posterior de Lord W. a la casa del detective demostraría que habría revuelto el departamento de la enfermera en busca de evidencias que lo comprometan, por lo que se dirigiría a su domicilio para recuperar el documento.

Los elementos son tan precarios que en un primer momento no podemos más que pensar en las consecuencias del paso del tiempo sobre el viejo detective. Pero una mirada crítica sobre los índices en juego nos permite también arriesgar otra hipótesis, que otro trabajo monográfico podría recuperar: Holmes, al decir de Tomatis que narraría Watson, "alternaba la cocaína y la ambición" (89) y "habría ido adoptando poco a poco ideas socialistas, incluso anarcosindicalistas" (100). El mismo Watson habría declarado "que Holmes una vez le habría dicho: ¿Qué se gana con defender el orden establecido...?” (100) ¿Acaso no es posible que el mismo Danny la Rata haya introducido el veneno en la maternidad y preparado el escenario, con sus sigilosos movimientos, para que finalmente Lord W. sea inculpado? ¿No es verosímil que la llegada de Lord W. al domicilio de Holmes responda precisamente a un telegrama enviado por la Rata para citarlo bajo cualquier pretexto colocando el veneno en el bolsillo del saco del Lord donde finalmente se lo encuentra? ¿El móvil? Precisamente la ideología de Holmes (manifestada ya en la defensa de un ladrón de mansiones burguesas) articulada en el grito que cierra el relato: “ $i \mathrm{La}$ horca estaría menos ocupada en sofocar a los hijos del pueblo si recibiese con más asiduidad las testas coronadas del pueblo!” (105).

25 Por lo que advertimos que Holmes ha modificado su relación con el futuro si recordamos que en el caso de Baskerville dice a Watson que "lo que una persona vaya a hacer en el futuro es muy difícil de prever". 
Advertimos entonces tres bordes que pueden fisurarse en los marcos de referencia: 1) los espacios internos; 2) los límites externos con otros textos; 3) el límite externo con la realidad.

La crítica policial, con su carácter intervencionista, apuesta a rastrear indicios a menudo más allá de los límites. Pero a ciencia cierta, el borde de un texto es siempre una problemática en la crítica literaria y el lugar dónde detenerse una mera arbitrariedad. Por ello mismo, en la medida en que hemos dicho que la crítica policial rastrea la narración de una alegoría de la lectura, podemos afirmar que se trata también de la historia del establecimiento, arbitrario aunque teóricamente fundado, de un marco de referencia.

\section{LO QUE DE VERDAD PASÓ EN DINAMARCA: LA REESCRITURA}

La segunda entrega de la trilogía de Bayard apunta a Hamlet (2003). Ya hemos indicado al inicio las incongruencias. Retomemos los dos problemas fundamentales.

En primer lugar, ¿por qué Claudio no reacciona ante la pantomima representada previamente donde se cuenta exactamente la historia del asesinato del rey de Dinamarca? Otra forma de encarar esta pregunta sería con otra: ¿por qué ha reaccionado frente a la obra principal representada? $\mathrm{La}$ respuesta, que Bayard retoma del shakesperiano Walter Wilson Greg, podría ser la siguiente: si Claudio reacciona a la obra de teatro no es porque sea el asesino (puesto que no ha reaccionado ante la pantomima), sino porque está cansado de las impertinencias de Hamlet.

En segundo lugar, ¿no es una coincidencia por lo menos extraña que haya existido una obra que Hamlet ya conocía donde se narra el asesinato de un rey según el modo en que el espectro le ha contado al príncipe?

Bayard procede entonces al segundo movimiento, con el que ya el lector se habrá familiarizado habiendo llegado hasta esta instancia: ¿quién, entonces, es el verdadero asesino? ¿No habrá sido acaso el mismo Hamlet, quien ha inspirado el modus operandi en aquella obra ya conocida, ha rechazado ese acto intolerable para su conciencia, ha alucinado la aparición de su padre y ha quitado así de su responsabilidad el peso del crimen? Hamlet, acusa Bayard, quien, cumpliendo una fantasía edípica, asesina a su padre y forcluye ese acto, poniendo en su lugar el delirio del fantasma que incrimina a su tío. 
¿Qué lee esta vez la crítica policial en Hamlet en la medida en que puede ser considerado un relato policial? A diferencia de los anteriores, el caso tomado aquí reúne la particularidad de haber sido enormemente trabajado por la crítica durante siglos, ofreciéndose como primera evidencia la multitud de divergencias en la interpretación. Este aspecto lleva a Bayard a compartir con el psicoanalista norteamericano Norman Holland una perspectiva similar frente a la obra de Shakespeare. Dice Bayard:

Entre dos personas que discuten sobre la obra de Shakespeare el objeto es sin duda idéntico, mientras se comparta la misma edición. Pero, aunque idéntico el objeto, el referente de sus discursos, es decir el mundo virtual poblado de criaturas imaginarias con las que mantienen diversas relaciones, es en cambio bien diferente. Entre el texto como objeto y el texto como referente se interpone la delgada hoja del lenguaje crítico, tejido por el imaginario, que separa al texto de sí mismo (Enquête 47).

Por su parte, afirma Holland que "si se observan los volúmenes sobre volúmenes de comentarios sobre Hamlet se advertirá que, finalmente, cada persona tiene su propio Hamlet" ("Hamlet - my greatest" 420 ). La frase de Holland proviene de un texto de 1975, titulado "Hamlet - my greatest creation", donde radicaliza en un ejercicio crítico un axioma ya anunciado en su libro 5 Readers Reading (1975b): la lectura es la recreación de la propia identidad.

En un sentido que continuaría la visión de Holland, el trabajo de Bayard sobre Hamlet expone la noción de paradigma interno y de reescritura de la obra analizada.

De este modo, volvemos a ampliar lo que queremos decir cuando la crítica policial lee una y otra vez la misma historia: la de la alegoría de la lectura. Es decir, el modo en que la lectura es aveuglante, el modo en que se establece un marco arbitrario de referencia y el modo en que se reescribe la propia identidad. En una palabra: el modo en que una carta llega siempre a su destino donde sea que es leída.

\section{BIBLIOGRAFÍA}

Barthes, Roland. S/Z. CEuvres complètes. Tome III. Paris: Seuil. 2002.

Bayard, Pierre. Qui a tué Roger Ackroyd ? Paris: Les Éditions de Minuit. 1998.

Enquête sur Hamlet. Le dialogue de sourds. Paris: Les Éditions de Minuit. 2002. 

2004.

Peut-on appliquer la littérature à la psychanalyse? Paris: Les Éditions de Minuit. L'Affaire du Chien des Baskerville. Paris: Les Éditions de Minuit. 2008.

Bloom, Harold. Cómo leer y por qué. Barcelona: Anagrama. 2007.

De Man, Paul. Blindness and Insigth. Essays in the Rethoric of Contemporary Criticism. Minneapolis: University of Minnesota Press. 2010.

Christie, Agatha. El asesinato de Roger Ackroyd. Buenos Aires: Planeta. 2007. The murder of Roger Ackroyd. New York: Harper Collins. 2011.

Conan Doyle, Arthur. The hound of the Baskervilles. London: Dover Thrift Edition. 1994. El sabueso de los Baskerville. Buenos Aires: Gradifco. 2007.

Derrida, Jacques. El concepto de verdad en Lacan. Buenos Aires: Homo Sapiens. 1977.

Felman, Shoshana. “De Sophocle à Japrisot (via Freud), ou pourquoi le policier ?". Littérature $\mathrm{N}^{\circ}$ 49. Le roman policier. (1983): 23-42.

Freud, Sigmund. La interpretación de los sueños en Obras completas. Tomo I. Madrid: Biblioteca Nueva. 1987.

Foucault, Michel. La verdad y las formas jurídicas. Barcelona: Gedisa. 2000.

Grégory, Fabien. Ce chien de Sherlock Holmes! Paris: Les éditions Chapitre.com. 2014.

Holland, Norman. "Hamlet - my greatest creation". Journal of the American Academy of Psychoanalysis № 3 (1975a): 419-427.

5 Readers Reading. New Haven and London: Yale University Press. 1975b.

Death in a Delphi Seminar. A postmodern mystery. New York: State University of New York Press. 1995.

“The Willing Suspension of Disbelief: A Neuro-Psychoanalytic View" PsyArt N ${ }^{o} .7$ (2003): http://www.psyartjournal.com/article/show/n_holland-the_willing_suspension_ of_disbelief_a_ne. Fecha de acceso: 16/09/2014.

Literatura, lectura y neuropsicoanálisis. Córdoba: Alción. 2015.

Iser, Wolfgang. "The Reading Process: a Phenomenological Approach". Reader-response criticism. Ed. Tompkins, Jane. Baltimore and London: The John Hopkins University Press, 1984. 50-69.

Johnson, Barbara. "The Frame of Reference: Poe, Lacan, Derrida. Yale French Studies No 55/56, Literature and Psychoanalysis. The Question of Reading: Otherwise (1977): 457-505.

Lacan, Jacques. Escritos 1. Buenos Aires: Siglo XXI. 2002.

Pastormerlo, Sergio. "Dos concepciones del género policial. Una introducción a la narrativa policial borgeana”. Literatura policial en la Argentina: Waleis, Borges, Saer. Ed. Néstor Ponce et al. La Plata: Universidad Nacional de La Plata, 1997. 17-44.

Poe, Edgar Alan. "La carta robada”. Obras. Tomo I. Barcelona: Ediciones Castell. 1985.

Saer, Juan José (2012) Lugar en Cuentos completos (1957-2000). Buenos Aires. Seix Barral.

Shakespeare, William. Hamlet. Obras completas. Tomo I. Madrid: Aguilar. 2003.

Sófocles. Tragedias. Madrid: Edaf. 2001. 
Wilson, Edmund. Who cares who killed Roger Ackroyd? Boston: Beacon Press. 1967.

Winnicot, Donald. Juego y realidad. Barcelona: Gedisa. 1994.

Žižek, Slavoj. ;Goza tu sintoma! Buenos Aires: Nueva Visión. 1994. 